\title{
sciendo Integrated sustainable freight transport system for city multi-floor manufacturing clusters
}

doi:10.2478/mape-2019-0015

Date of submission to the Editor: 05/2018

Date of acceptance by the Editor: 07/2018

MAPE 2019, volume 2, issue 1, pp. 151-160

\section{Bogusz Wiśnicki}

ORCID ID: 0000-0003-2932-8969

Tygran Dzhuguryan

ORCID ID: 0000-0001-5613-9448

Maritime University of Szczecin, Poland

\section{INTRODUCTION}

The purpose of the article is to present an innovative freight transport system dedicated for city clusters. A number of initial assumptions regarding the functioning of urban clusters and the logistics system that supports them are adopted. It is assumed that in large agglomerations clustered areas will be separated, where spatially limited small-scale production will be possible. Such needs go against the multi-floor manufacturing technology. Necessary complement to the cluster network (coexistence of several clusters in large urban areas) is a tailored transport and logistics system (Azapagic \& Perdan, 2000; Sikdar, 2003; Sala et al., 2015; Kluczek, 2016). Figure 1 shows the network of industrial clusters were multi-floor manufacturing companies are located and dedicated logistics system. Mega cluster (1) includes medium (2) and small (3) industrial clusters with logistics nodes (4) that form the city logistics cluster (5). The logistics nodes are well connected to available transport infrastructure, including: road (6), railway (7) and river (8) systems.

The main functionality of the system is carriage of small and medium size cargo units in the urban area. This freight transport system must perform just-in-time supply and distribution chains, while meeting two very important efficiency requirements. First is technological integration and second is high-sustainability (Sala et al., 2015; Anschutz et al., 2004).

Technological integration is extremely demanding due to the need to combine internal (cargo lifts, conveyers, trolleys) and external (e-trucks, railway buses, barges) transport systems, which are normally not connected, and in conditions of restrictions imposed by urban high-density housing. Efficiency criteria for sustainable transport are low emission $\left(\mathrm{CO}_{2}, \mathrm{NO}_{x}, \mathrm{PM}\right.$, noise), waste management, low accident rate and high-utilization of cargo capacity (Sikdar, 2003; Sarkis \& Zhu, 2018).

Additionally, the logistics system must integrate the various stages of the logistics process, differing in customer expectations and external constraints (Khorram Niaki and Nonino, 2017). The differences concern internal transport carried out inside the building, urban transport and long-distance transport with the use of intermodal transport means.

\footnotetext{
•b.wisnicki@am.szczecin.pl
} 


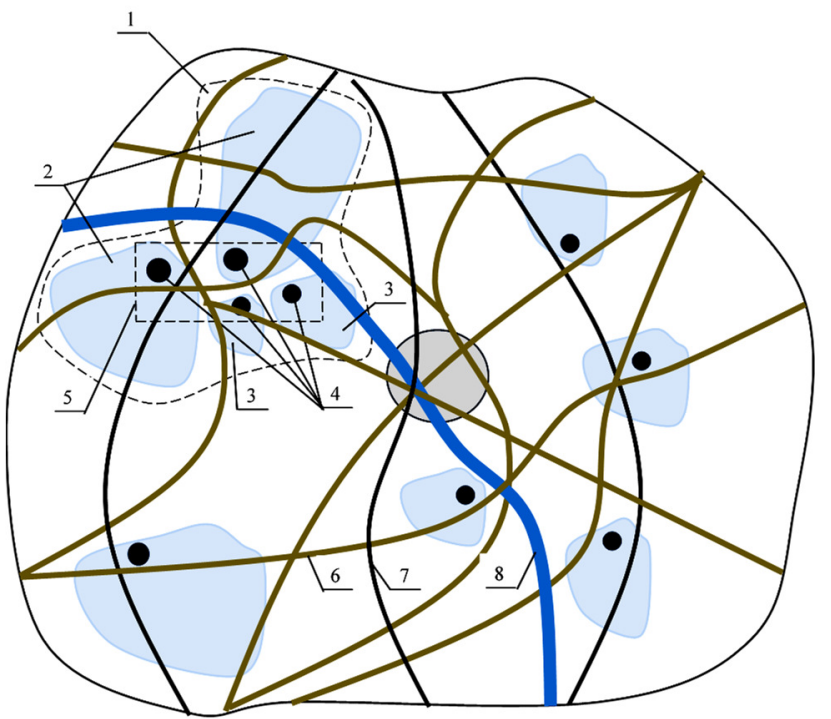

Fig. 1 Network of industrial clusters and accompanying logistics system

Integrated sustainable freight transport system in urban area is challenging and one of key solution proposed by authors is innovative transport unit, named Intelligent Reconfigurable Trolleys (IRTs). These cargo trolleys are designed to facilitate shipment of freight within large agglomerations where industry clusters with multi-floor manufacturing facilities are located.

The following part will present the concept of multi-floor manufacturing with explanation with special attention will be to in-house transport servicing various companies located in one building. The next chapter will describe the concept of the Intelligent Reconfigurable Trolleys (IRTs) including proposed variants of construction and equipment. Finally, the calculation model of integrated sustainable freight transport system with the use of IRTs will presented.

\section{CONCEPT OF MULTI-FLOOR MANUFACTURING (MFM)}

The trends of the urbanization are associated with providing of a green manufacturing and development of the concept of effective work in the urban environment (Fujita \& Thisse, 2002; Westkämper, 2014; Spath D. et al., 2017). This in turn is closely related to efficient logistics and mobility, improvement of the transport communication and creation of innovative technological equipment and products for the logistics system users (Dzhuguryan \& Jóźwiak, 2016). Under these conditions, the city multi-floor manufacturing and city logistics with multi-floor warehouses in the residential areas of big cities are developed. The location of multi-floor manufacturing buildings directly in the residential area of a big urban agglomeration enforces the necessity of use "clean technologies" for products manufacturing. It is obvious that the enterprises of metallurgical and chemical industry, as well as heavy and medium-sized machinery are located in industrial areas away from inhabited areas. However, part "clean" components for such industries can be produced by city multi-floor manufacturing (Dzhuguryan at al., 2018b).

Multi-floor manufacturing (MFM) (Figure 2) is a multi-story building (1) with freight elevators (2), in the floors of which manufacturing of products for the population of the huge city are organized. 


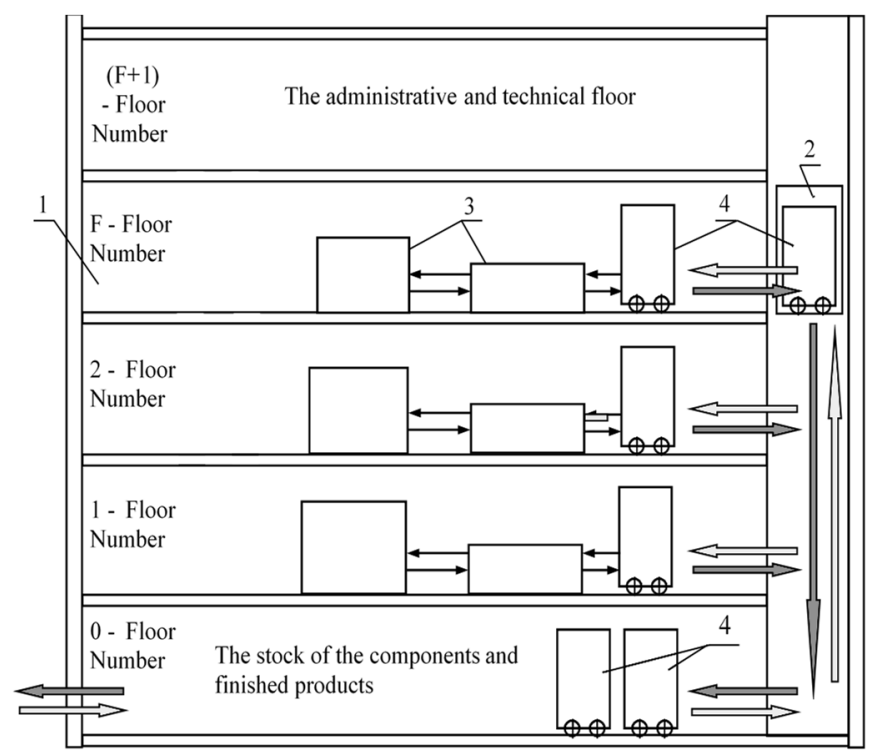

Fig. 2 Multi-floor manufacturing

Sourtce: (Dzhuguryan et al., 2018a).

On the ground floor and the upper floors are respectively the main stock and administrative and technical premises. On the other floors technological equipment (3) for manufacturing is installed. Delivery of materials and components for production on floors and shipment of finished products to the main stock are carried out by means of trolleys (4) and freight elevators (Dzhuguryan \& Jóźwiak, 2016). It is important to use trolleys in flexible production as a tool of implementation of the "Kanban Cards" philosophy (Sodkomkham \& Chutima, 2016) in the MFM, which reduces the storage time of freights and finished goods in warehouses and shortens the time of receipt of goods and services by customers.

The located group of MFM on some territory of major cities that often interconnected by production networks, can be integrated into clusters. The number of MFM clusters in the major city depends on its size and economic expediency. The infrastructure of MFM cluster includes production and non-production (social) infrastructure with different form of ownership that is necessary for its efficient functioning. Especially, there should be noted a logistics infrastructure that is designed for maintenance of systems of procurement, delivery, storage and supply of products to the consumer.

\section{CONCEPT OF INTELLIGENT RECONFIGURABLE TROLLEYS (IRTS)}

The Intelligent Reconfigurable Trolleys (IRTs) concept is a system of provisions defining the creation of an innovative transport designed to serve MFM in the urban environment. The main provisions of the IRTs concept are as follows (Dzhuguryan et al., 2018c):

- increase the level of transport services for MFM,

- simple and rapid transformation for the transport of new freights in flexible manufacturing conditions,

- transportation by various means of transport including: cranes, conveyors, semielectric power trucks, freight elevators, pallet jack trucks, light and medium trucks, intermodal transport etc.,

- registration, certification, real-time identification and monitoring of IRTs,

- supply chain management in different levels of the manufacturing organization. 
The intelligent reconfigurable trolleys considering their adaptation to the user's needs can be in different structural configurations. Four basic construction types can be distinguished:

- general IRT - for storage and transportation of solid-state cargo without and with packaging,

- bulk IRT - for storage and transportation of bulk cargo,

- tank IRT - for storage and transportation of liquids,

- waste IRT - for storage and transportation of manufacturing and domestic waste.

Regardless of IRT type, they are all built from the basic components: load-carrying modular frames, functional modular frames and supporting frame with wheels (Wiśnicki \& Dzhuguryan, 2018). In addition to basic components there are operational and fleet ones. Operational components are closely related to the adaptation to the transported cargo and the desired functionalities and fleet components are necessary for connection, reloading and transport of IRTs in the dedicated transport system.

Selecting or changing the design of IRT is carried out by its owner, considering the market demand for this type of transportation services. The IRTs fleet operator can provide information to clients about the market needs for transport services.

Each of the possible IRT design variants is assigned the IRT code. The IRT code is stored in the database of IRTs fleet operator and is used in all data transitions generated by identification and monitoring device. The stored and transmitted data can be easily extended by information about IRT's owner, freight data, safety instruction, etc. This is the basis for IRT real-time identification and monitoring. There are several tracking systems available to use: GPS, NFC, GTIN, RFID, Barcode and all loT compatible systems (Christopher, 2011).

Fleet of IRTs can be the core element of the logistics system offering high-quality and cost-effective deliveries. The logistics operator managing the IRT fleet (IRTs fleet operator) should implement all actions to ensure the appropriate number and type of IRTs to meet the needs of all supply chain participants. It is important in the supply chain to avoid downtime of IRTs and timely delivery of goods with minimal costs. In this case, it is also necessary to consider the loss of time for the re-equipment, repair of the IRTs before their recycling.

IRTs with freight are delivered to the main stock at the ground floor of MFM buildings and there are sorted and formed into multi-IRTs (Figure 1). The main group is delivered to the nearest city logistics node and in justified cases IRTs or multi-IRTs are transported to other destinations within the same cluster. In city logistics nodes, IRTs are again sorted into two groups. In urban logistics nodes, IRTs are sorted into several groups. One group is delivered to Intermodal Transport Terminal, servicing interregional deliveries withy the use of rail or inland shipping transport. Remaining groups are delivered to other city logistics nodes within the same agglomeration.

Figure 3 shows a logistics system within MFM cluster:

1 - city MFM cluster;

2 - MFM buildings;

3 - logistics node;

4 - internal freight transport links of the cluster;

5 - external freight transport links of the cluster. 


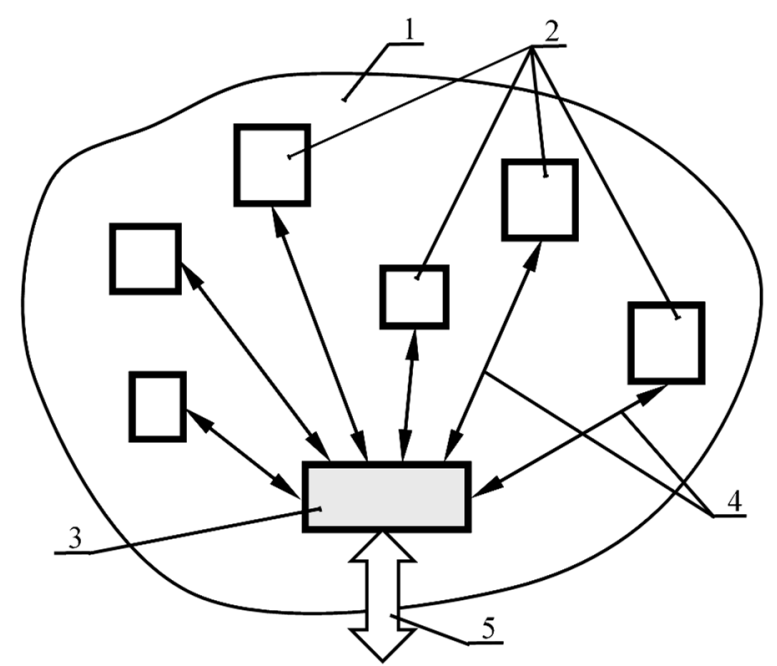

Fig. 3 Logistics system of multi-floor manufacturing cluster

\section{MODEL OF INTEGRATED TRANSPORT SYSTEM FOR MFM CLUSTER}

According to the adopted criteria of the efficiency of the transport system, i.e. technological integration and high-sustainability, the freight transport system is proposed dedicated for MFM clusters. This complex logistic solution integrates elements (freight elevators, IRTs, loading and unloading devices, e-trucks, trams) which meet the requirements of environmentally sustainable development. It means the proposed integrated transport system guarantee sustainable operation of the city MFM cluster.

Below, a mathematical model describing logistics processes within the city MFM cluster area is presented. The model allows for the quantitative matching of key elements of the transport system (freight elevators, IRTs, e-trucks) to the MFM cluster needs. As a result, you can minimize system losses and reduce the negative impact of transport on the environment.

The transport process at the MFM buildings begins with vertical transport using freight elevators. The freight elevators consistently stop on each floor with loading and unloading the same number of the IRTs. In this case, the round-trip time $T_{R}$ of the freight elevator for i-th MFM building can be found from the following expression [1]:

$$
T_{R . i}=\frac{K_{C . i} f_{i} F_{i}\left(F_{i}+1\right)}{3600 K_{E F . i} K_{E i} v_{E . i}}
$$

where:

$T_{R . i}$ - the freight elevator round trip time for i-th MFM building, h;

$f_{i}$ - the interfloor distance for i-th MFM building, $\mathrm{m}$;

$v_{E . i}$ - the rate speed of the freight elevator for $\mathrm{i}$-th MFM building, $\mathrm{m} / \mathrm{s}$;

$F_{i}$ - the number of floor of the manufacturing part of the i-th MFM building;

$k_{C . i}-$ the coefficient of operation time cycle losses for the freight elevators of the i-th MFM building;

$K_{E F . i}$ - the coefficient of the freight elevator occupancy rate;

$K_{E . i}$ - the coefficient taking into account the average weekly downtime of freight elevators falling on their round trip time.

The coefficient of operation time cycle losses for the freight elevators of the i-th MFM building is find: 


$$
K_{C . i}=1+\frac{v_{E . i}\left[4 \lambda_{E . i} t_{E . i}+2\left(2 t_{f . i}+t_{o . i}+t_{c . i} t_{m p . i}\right)\right]}{f_{i}\left(1+F_{i}\right)}
$$

where:

$t_{E . i}$ - the IRT loading/unloading time for i-th MFM building, s;

$t_{f . i}-$ the single floor flight time representing the time of acceleration and deceleration for i-th MFM building, s;

$t_{0 . i}$ - the door opening time for i-th MFM building, s;

$t_{c . i}$ - the door closing time for i-th MFM building, s;

$t_{m p . i}$ - the time of the multi-IRT handling operations in the ground floor area for i-th MFM building, s;

$\lambda_{\mathrm{E} . \mathrm{i}}$ - the number of the IRTs in the freight elevator in the i-th MFM building, pcs.

During the round-trip time $T_{R T}$ of freight elevators in the picking area on the ground floor of the i-th manufacturing building the following number of the IRTs with various goods are shipped:

$$
\lambda_{R . i}=F_{i} \lambda_{E . i} \varepsilon_{i}
$$

and the performance of the i-th MFM building are determined as:

$$
P_{i}=\frac{F_{i} \lambda_{E . i} \varepsilon_{i}}{T_{R . i}}
$$

where:

$\lambda_{R . i}$ - the number of the IRTs in the picking area of the i-th manufacturing building during the round trip time of freight elevators, pcs;

$\varepsilon_{i}$ - the number of the freight elevators in the i-th MFM building, pcs;

$P_{i}$ - the performance of $\mathrm{i}$-th MFM building, IRTs/h.

The downtime of the separate technology equipment in calculations of the performance of the MFM building is not considered because it can be compensated by increase in productivity of other technology equipment.

The performance of the i-th manufacturing building should correspond to the capacity transport system of the city MFM cluster:

$$
P_{i}=C_{i}=\frac{N_{T . i} \lambda_{T . i}}{T_{T . i}}
$$

where:

$C_{i}$ - the capacity of the transport serving for i-th MFM building, IRTs/h;

$\lambda_{T, i}$ - the number of the IRTs in the e-truck for servicing of the i-th MFM building, pcs;

$N_{T, i}$ - the number of the e-truck for servicing of the i-th MFM building, pcs;

$T_{T, i}$ - the e-truck round trip time between i-th MFM building and a logistics node of the cluster, $\mathrm{h}$.

The round trip time $T_{T . i}$ of the e-truck between i-th MFM building and the logistics node of the cluster can be found from the following expression:

$$
T_{T . i}=\frac{2}{K_{T F . i} K_{T . i}}\left(\frac{L_{i}}{v_{T . i}}+\frac{2 \lambda_{T . i} t_{T . i}}{3600}\right)
$$

where:

$L_{i}-$ the distance between i-th MFM building and the logistics node of the cluster, $\mathrm{km}$; $v_{T, i}$ - the rate speed of the e-truck in the city MFM cluster, $\mathrm{km} / \mathrm{h}$;

$t_{T . i}$ - the IRT loading/unloading time for the e-truck i-th MFM building, s;

$K_{T F . i}$ - the coefficient of the e-truck occupancy rate for the i-th MFM building; $K_{T . i}-$ the coefficient taking into account the average weekly downtime of e-truck falling on 
their round trip time for the i-th MFM building.

Based on the obtained equations, we will determine the need for the i-th MFM building in the transport fleet, which would ensure the production of supplies with a given reliability:

$$
\begin{gathered}
N_{T . i}=\frac{P_{i} T_{T . i}}{\lambda_{T . i}} \\
\lambda_{i}=\frac{2 N_{T . i} \lambda_{T . i}}{K_{\lambda . i}}
\end{gathered}
$$

where:

$N_{T . i}$ - the number of the e-trucks for the i-th MFM building;

$\lambda_{i}$ - the number of the IRTs for the i-th MFM building;

$K_{\lambda . i}$ - the coefficient of the IRTs using for the i-th MFM building.

The values of the coefficients can be obtained based on statistical processing of data on the operation of the freight elevators, e-trucks and IRTs with a given probability, which allows to determine their guaranteed values with a given accuracy and reliability. As an example, consider the sequence of actions to determine the value of the coefficient $K_{\lambda . i}$ for the i-th MFM building within $n$ weeks.

At the first stage of statistical research we find a data set of the coefficient $K_{\lambda . i}$ within $\mathrm{n}$ weeks, which will take the following form:

$$
K_{\lambda . i .1}=\frac{T_{i .1}-T_{r . i .1}}{T_{i .1}}, \ldots K_{\lambda . i .1}=\frac{T_{i . n}-T_{r . i . n}}{T_{i . n}}
$$

where:

$K_{\lambda . i .1}, K_{\lambda . i . n}$ - accordingly, the coefficients of the IRTs using on the first and on the $\mathrm{n}$ week for the i-th MFM building;

$T_{i .1}, T_{i . n}$ - accordingly, the total operation time of the IRTs on the first and on the $\mathrm{n}$ week for the i-th MFM building;

$T_{\text {r.i. } 1}, T_{\text {r.i.n }}$ - accordingly, the downtime of the IRTs on the first and on the $\mathrm{n}$ week for the i-th MFM building.

At the second stage are used the standard statistics research for this data set. Given the large number of independent factors that affect the values of the coefficient $K_{\lambda . i}$ it is possible to propose the hypothesis of normality data set. If the hypothesis is confirmed by normality tests (for example, Shapiro - Wilk test, Kolmogorov test or Pearson's chi-squared test), the guaranteed value of the coefficient $K_{\lambda . i}$ with a given accuracy $(0,95 ; 0,99)$ and reliability $(0.95 ; 0.99 ; 0.999)$ of assessment can be determined by the equation:

$$
K_{\lambda . i}=\left(\frac{1}{n} \sum_{j=1}^{n} \frac{T_{i . j}-T_{i . r, j}}{T_{i . j}}\right)-l_{\lambda . i} \sigma_{\lambda . i}
$$

where:

$\sigma_{\lambda . i}$ - the standard deviation of the coefficient $K_{\lambda . i}$ for the i-th MFM building;

$l_{\lambda . i}$ - the mean of standard deviation of the coefficient $K_{\lambda . i}$ with a given accuracy and reliability of assessment for the i-th MFM building;

$n$ - the number of weeks for the statistical research for the i-th MFM building.

Similarly, the guaranteed values and other coefficients are found: 


$$
\begin{gathered}
K_{E F . i}=\left(\frac{1}{n} \sum_{j=1}^{n} \frac{\lambda_{E F . i . j}-\lambda_{E F . i . f, j}}{\lambda_{E F . i . j}}\right)-l_{E F . i} \sigma_{\lambda E F . i} \\
K_{E . i}=\left(\frac{1}{n} \sum_{j=1}^{n} \frac{T_{E . i . j}-T_{E . i . r . j}}{T_{E . i . j}}\right)-l_{E . i} \sigma_{E . i} \\
K_{T F . i}=\left(\frac{1}{n} \sum_{j=1}^{n} \frac{\lambda_{T F . i . j}-\lambda_{T F . i . f, j}}{\lambda_{T F . i . j}}\right)-l_{T F . i} \sigma_{T F . i} \\
K_{T . i}=\left(\frac{1}{n} \sum_{j=1}^{n} \frac{T_{T . i . j}-T_{T . i . r . j}}{T_{T . i . j}}\right)-l_{T . i} \sigma_{T . i}
\end{gathered}
$$

where:

$\lambda_{E F . i . j}$ and $\lambda_{E F . i . f . j}$ - accordingly, the total and actual number of the IRTs that could carry the freight elevators in j-th weeks for the i-th MFM building;

$\lambda_{\text {TF.i.j }}$ and $\lambda_{\text {TF.i.f.j }}$ - accordingly, the total and actual number of the IRTs that could carry the e-trucks in j-th week for the i-th MFM building;

$T_{E . i . j}$ and $T_{T . i . j}$ - accordingly, the total operation time of the freight elevators and etrucks in $\mathrm{j}$-th week for the i-th MFM building;

$T_{E . i . r . j}$ and $T_{T . i . r . j}$ - accordingly, the downtime of the freight elevators and e-trucks in $\mathrm{j}$-th week for the i-th MFM building;

$\sigma_{E F . i}, \sigma_{E . i}, \sigma_{T F . i}, \sigma_{T . i}$ - accordingly, the standard deviation of the coefficients $K_{E F . i}$, $K_{E . i}, K_{T F . i}, K_{T, i}$ for the i-th MFM building;

$l_{E F . i}, l_{E . i}, l_{T F . i}, l_{T . i}$ - accordingly, the mean of standard deviation of the coefficients $K_{E F . i}, K_{E . i}, K_{T F . i}, K_{T . i}$ with a given accuracy and reliability of assessment for the i-th MFM building.

In this case, the reliability of the assessment with a given accuracy for the transport system of the i-th MFM building can be found as:

$$
R_{i}=R_{\lambda . i}, R_{E F . i} R_{E . i} R_{T F . i} R_{T . i}
$$

where:

$R_{i}$ - the reliability of the assessment with a given accuracy for the transport system of the i-th MFM building;

$R_{\lambda . i}, R_{E F . i}, R_{E . i}, R_{T F . i}, R_{T . i}$ - accordingly, the reliabilities of the assessment with a given accuracy of the obtained values of the coefficients $K_{\lambda . i}, K_{E F . i}, K_{E . i}, K_{T F . i}, K_{T . i}$. At the third stage, we check the hypotheses of homogeneity of the samples of the corresponding coefficients for all MFM buildings of the cluster using the statistical Kolmogorov-Smirnov test. This allows us to test the hypothesis that independent samples belong to the same distribution law. If the test confirmed the hypothesis of homogeneity of samples for each MFM buildings, it is possible to find the values of these coefficients $K_{\lambda}, K_{E F}, K_{E}, K_{T F}, K_{T}$ for the cluster as a whole:

$$
K_{\lambda}=\sum_{i=1}^{k} K_{\lambda . i} ; K_{E F}=\sum_{i=1}^{k} K_{E F . i} ; K_{E}=\sum_{i=}^{k} K_{E . i} ; K_{T F}=\sum_{i=1}^{k} K_{T F . i} ; K_{T}=\sum_{i=1}^{k} K_{T . i}
$$

where:

$k$ - the number of the MFM buildings in the cluster.

Based on the obtained equations, it is possible to determine the need of the city MFM cluster in the transport fleet and assess the reliability of its operation with a given accuracy: 


$$
\begin{gathered}
N_{T}=\sum_{i=1}^{k} \frac{P_{i} T_{T . i}}{\lambda_{T . i}} \\
\lambda=2 \sum_{i=1}^{k} \frac{2 N_{T . i} \lambda_{T . i}}{K_{\lambda . i}} \\
R=\prod_{i=1}^{k} R_{\lambda . i} R_{E F . i} R_{E . i} R_{T F . i} R_{T . i}
\end{gathered}
$$

where:

$N_{T}$ - the number of the e-trucks for the city MFM cluster;

$\lambda$ - the number of the IRTs for the city MFM cluster;

$R$ - the reliability of the assessment with a given accuracy for the transport system of the city MFM cluster.

The coefficients $K_{\lambda}, K_{E F}, K_{E}, K_{T F}, K_{T}$ and the reliability parameter of transport operations can be used to assess the efficiency of the elements of the transport system of the city MFM cluster.

\section{CONCLUSIONS}

The presented concept of integrated sustainable freight transport system is the result of a broader study on the effectiveness of production and transport systems in urban areas. Research includes core process areas: multi-floor manufacturing, city manufacturing clusters, city logistics nodes, agglomeration transport network, intermodal transport terminal. These crucial points and connections between them are covered the supply chain management system adapted to the needs of big cities that want to keep their production functions and at the same time meet the requirements of sustainable transport.

The integrated sustainable freight transport system described in this paper is dedicated to multi-floor manufacturing clusters and therefore meets two efficiency requirements: technological integration and process sustainability. The integration of in-house transport system servicing multi-floor manufacturing building and supply chain deliveries to/from logistic node is based on efficient use of Intelligent Reconfigurable Trolleys (IRTs). Hence, the calculation model of integrated freight transport system with the use of IRTs was presented. The model allows for the quantitative matching of key elements of the transport system to the multi-floor manufacturing cluster needs. Quantitatively selected and functionally integrated elements of proposed transport system allows to minimize losses and reduce the negative impact of transport on the environment.

\section{REFEENCES}

Anschutz, J., IJgosse, J. and Scheinberg, A. (2004). Putting Integrated Sustainable Waste Management into Practice Using the ISWM Assessment Methodology: ISWM Methodology as Applied in the UWEP Plus Programme (2001-2003), WASTE: Gouda, The Netherlands.

Azapagic, A. and Perdan, S. (2000). Indicators of sustainable development for industry: a general framework, Process Saf Environ Prot, 78(4), pp. 243-261.

Christopher, M. (2011). Logistics \& Supply Chain Management: creating value-adding networks, Pearson Education Limited, pp. 9-10. 
Dzhuguryan T. and Jóźwiak Z. (2016). Infrastructure for Multi-Floor Virtual Enterprises System, Systemy wspomagania w inżynierii produkcji, P.A. Nowa S.A. Gliwice, 3(15), pp. 70-78.

Dzhuguryan, T., Jóźwiak, Z., Deja, A. and Semenova, A. (2018a). Infrastructure and Functions of a City Logistics Node for Multi-Floor Manufacturing Cluster, 8th International Scientific Conference CMDTUR 2018, Žilina, Slovakia, pp. 196-201.

Dzhuguryan, T., Wiśnicki, B. and Jóźwiak, Z. (2018b). Modular Loading Units for Facilitating Multi-Floor Manufacturing and City Logistics, Scientific Journals of the Maritime University of Szczecin, 55(127), pp. 73-78.

Dzhuguryan, T., Wiśnicki, B. and Dudek, T. (2018c). Concept of Intelligent Reconfigurable Trolleys for City Multi-Floor Manufacturing and Logistics System, 8th Carpathian Logistics Congress, Prague, Czech Republic.

Fujita, M. and Thisse, J.-F. (2002). Economics of Agglomeration: Cities, Industrial Localization, and Globalization, Cambridge, Massachusetts: Cambridge University Press.

Khorram Niaki, M. and Nonino, F. (2017). Additive manufacturing management: a review and future research agenda. International Journal of Production Research, 55(5), pp. 14191439.

Kluczek, A. (2016). Application of Multi-criteria Approach for Sustainability Assessment of Manufacturing Processes. Management and Production Engineering Review, 7(3), pp. 62-78.

Sala, S., Ciuffo, B. and Nijkamp, P. (2015). A systemic framework for sustainability assessment. Ecological Economics, 119, pp. 314-325.

Sarkis, J. and Zhu, Q. (2018). Environmental sustainability and production: taking the road less travelled, International Journal of Production Research, 56:1-2, pp. 743-759.

Sikdar, SK. (2003). Sustainable development and sustainability metrics, AIChE J 49(8), 19281932.

Sodkomkham, T. and Chutima, P. (2016). Lean Six Sigma Application in Rear Combination Automotive Lighting Process. IOP Conference Series: Materials Science and Engineering, 131(1).

Spath D. et al. (2017). Entwicklungen in der Unternehmensorganisation, Springer, Berlin.

Westkämper E. (2014). Towards the Re-Industrialization of Europe. A Concept for Manufacturing for 2030, Berlin: Springer, Germany.

Wiśnicki, B. and Dzhuguryan, T. (2018). Flexible Logistic Processes with the Use of Modular Loading Units, Technologia i automatyzacja montażu, 2, pp. 16-20.

Abstract. The purpose of the article is to present the integrated sustainable freight transport system dedicated for city clusters. The presented concept and calculation model is the result of a broader study on the effectiveness of production and transport systems in urban areas. The integrated sustainable freight transport system described in this paper serves multi-floor manufacturing clusters and therefore meets two efficiency requirements: technological integration and process sustainability. The integration of in-house transport system servicing multi-floor manufacturing building and supply chain deliveries to/from logistic node is based on efficient use of Intelligent Reconfigurable Trolleys (IRTs). The calculation model of integrated freight transport system allows for the quantitative matching of key elements of the transport system to the multi-floor manufacturing cluster needs. Properly selected and functionally integrated elements of proposed transport system allows to minimize losses and reduce the negative impact of transport on the environment.

Keywords: multi-floor manufacturing, transport system, supply chain management 